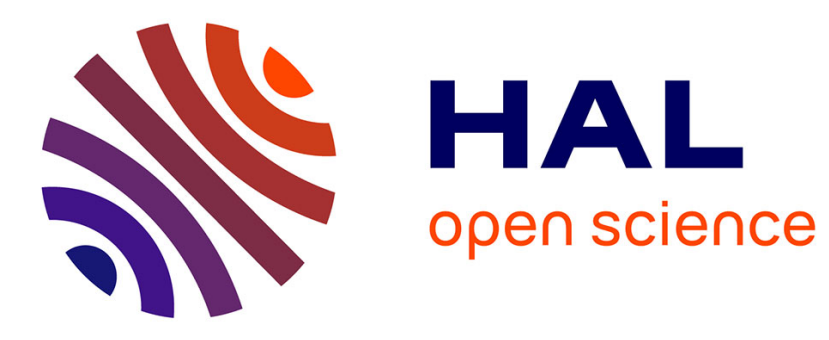

\title{
Le GÉVA Sco. Entre suivi personnalisé et harmonisation des pratiques évaluatives
}

Martial Meziani, Roberto Toledo, Hugo Dupont, Séverine Mayol

\section{To cite this version:}

Martial Meziani, Roberto Toledo, Hugo Dupont, Séverine Mayol. Le GÉVA Sco. Entre suivi personnalisé et harmonisation des pratiques évaluatives. La nouvelle revue de l'adaptation et de la scolarisation, 2016, Vers une évaluation scolaire inclusive?, 74, pp.145-158. 10.3917/nras.074.0145 . hal-01836959

\section{HAL Id: hal-01836959 \\ https://hal.science/hal-01836959}

Submitted on 22 Dec 2021

HAL is a multi-disciplinary open access archive for the deposit and dissemination of scientific research documents, whether they are published or not. The documents may come from teaching and research institutions in France or abroad, or from public or private research centers.
L'archive ouverte pluridisciplinaire HAL, est destinée au dépôt et à la diffusion de documents scientifiques de niveau recherche, publiés ou non, émanant des établissements d'enseignement et de recherche français ou étrangers, des laboratoires publics ou privés. 


\title{
Le GÉVA Sco \\ Entre suivi personnalisé et harmonisation des pratiques évaluatives
}

\author{
Martial MEZIANI \\ Chargé de recherche, INS HEA, Grhapes (EA 7287) \\ Roberto TOLEDO \\ Docteur en philosophie, Grhapes (EA 7287) \\ Hugo DUPONT \\ Maître de conférences, Université de Poitiers, Gresco (EA 3815) \\ Séverine MAYOL \\ Docteure en sociologie \\ Université Paris Descartes, LASCO - Mines Telecom
}

Résumé: De nos jours, les usagers sont poussés à participer à l'évaluation de leurs propres besoins. Cette participation s'inscrit dans les demandes relatives au droit à la compensation en matière d'accès à l'école des élèves reconnus handicapés par les MDPH. Le GÉVA Sco, guide d'aide à l'évaluation des besoins de ces élèves, s'inscrit dans une logique d'optimisation du suivi personnalisé par l'harmonisation des décisions au niveau national. Cet article, tiré d'une recherche portant sur cet outil, donne à voir les différents registres mobilisés et leurs réagencements liés à son apparition afin de décider l'attribution des aides à destination des élèves handicapés. Les résultats montrent que le jugement rendu et le sentiment de justice s'appuient sur l'articulation de dimensions subjectives, fondées sur l'empathie, avec d'autres objectives, présentes dans les différents bilans et diagnostics.

Mots-clés: Éducation inclusive - Évaluation des besoins - Justice sociale - MDPH - Politiques sociales Scolarisation.

\section{The GÉVA Sco. Between personalized follow-up and harmonization of practical assessment}

Summary: Welfare policies push users to participate into assessment of their own needs, particularly in the access to school for disabled people. GÉVA Sco is a guide to assess special needs of pupils with disabilities. Through this tool, it is to optimize personalized follow-up by harmonization of decisions in France. This article, based on a research about the GÉVA Sco, shows the categories used to judge and their rearrangement with the apparition of this tool. The analysis shows that the judgement rendered and sense of justice are founded on the association of subjective and objective dimensions, based on empathy and different diagnosis.

Keywords: Inclusive education - Moral economy - Needs assessment - Schooling - Social justice - Welfare policy. 
E GÉVA Sco, Guide d'aide à l'évaluation de l'autonomie des élèves reconnus handicapés, s'inscrit dans une logique visant à la fois à favoriser l'harmonisation des décisions sur l'ensemble du territoire hexagonal (CNSA, 2012 ; Amara et al., 2012) et à optimiser l'accompagnement individuel des élèves (Blanc, 2002; Barreyre, Peintre, 2004). Dans cette perspective, les acteurs, qu'ils soient sur le terrain ou dans les $\mathrm{MDPH}^{1}$, doivent suivre au plus près les besoins individuels et évaluer selon des critères qui soient les mêmes pour tous, afin d'obtenir un sentiment de justice. S'inscrivant dans le cadre réglementaire, le GÉVA Sco intègre une évaluation pluridisciplinaire effectuée par des spécialistes détenteurs de savoirs spécifiques relatifs au travail social, à la médecine, à la psychologie, au monde de l'éducation et à l'administration. Par cet intermédiaire, le but est d'obtenir une vision globale de la situation de la personne, tout en tenant compte de la parole des usagers (CNSA, 2010), via différents éléments, dont le GÉVA Sco. À travers ce document, il s'agit de changer le regard porté par les enseignants sur les élèves reconnus handicapés, en les incitant à inscrire leur évaluation scolaire dans un cadre plus large (CNSA, 2012). L'évaluation pluridisciplinaire pousse les acteurs à une forme de coopération relevant de l'expertise de consensus (Théry, 2005), dans laquelle le regard des acteurs de terrain vaut de manière égale (Lesain-Delabarre, 2006; Tessaire, 2007). Autrement dit, les membres de l'équipe pluridisciplinaire d'évaluation sont censés considérer de la même manière les savoirs professionnels et les savoirs expérientiels des familles, comme c'est le cas dans d'autres domaines (Epstein, 1995; Akrich, Rabeharisoa, 2012). Cette forme d'expertise, fondée à partir du croisement de plusieurs expertises, se répète régulièrement dans le temps et produit des normes, relevant de l'expertise instituante (Castel, 1991). Se constitue alors des registres visant à évaluer l'ensemble des situations étudiées.

Cet article, tiré d'une recherche ${ }^{2}$ relative à l'usage du GÉVA Sco, porte sur ces registres mobilisés dans un cadre légal confrontant les acteurs à des doubles contraintes (Bateson, 2008), entre suivi personnalisé et harmonisation des pratiques. Pour réaliser l'analyse, nous nous sommes appuyés sur plusieurs auteurs ayant travaillé sur les registres mobilisés dans différentes commissions d'attributions d'aides sociales. Fassin $(2000,2010)$, dans une recherche sur les stratégies rhétoriques utilisées dans les courriers de demandes d'aide d'urgence, a repéré l'utilisation de plusieurs registres relevant de la compassion, de la nécessité, de la justice et du mérite. Nous nous sommes également appuyés sur les travaux de Bureau et Rist (2013) qui ont étudié les auditions d'usagers en Commissions des droits à l'autonomie (CDA) et en particulier les principaux registres d'expression mobilisés pour expliquer leur situation. Dans leur étude, elles ont consigné quatre registres: la mise en scène du handicap, I'argumentation à partir de données factuelles, I'appel au sens moral et la revendication d'une reconnaissance du mérite (Bureau et Rist, 2013).

Dans notre enquête, nous nous sommes intéressés aux registres mobilisés par l'ensemble des acteurs concourant à la scolarisation. Les propos tenus par les différents acteurs lors des différentes étapes ne peuvent donc pas être compris de la même

1. Maison départementale des personnes handicapées.

2. Cette recherche a été financée par I'INS HEA et la Caisse nationale de solidarité pour l'autonomie. Cette recherche a également été menée sous la responsabilité scientifique de S. Ebersold 
manière, dans la mesure où les acteurs, ainsi que les différentes réunions étudiées n'ont pas la même fonction que les registres étudiés par Fassin (2000) ou Bureau et Rist (2013). Dans ces travaux, les auteurs ont étudié les stratégies rhétoriques des usagers, tandis qu'ici nous nous attelons à la construction de la décision entre différents acteurs, tels que les parents, les enseignants, les agents MDPH et les membres des $\mathrm{CDAPH}^{3}$. En ce sens, ils portent des intérêts potentiellement divergents et n'inscrivent pas leur action dans une seule et même fonction.

Ainsi, dans le cadre de notre enquête, les registres mobilisés lors des différentes réunions sont des conventions (Boltanski, Thévenot, 1991) que l'ensemble des acteurs ont mis au point, généralement à partir de critères relatifs à la solidarité d'État. Pour les professionnels en question, l'enjeu est de traduire (Latour, 1989; Bureau, 2013) des dimensions relevant de la justesse de l'évaluation et du sentiment de justice en droits effectifs, notamment par des dimensions éthiques, juridiques et économiques. Ce processus de traduction se joue non seulement sur la finalité de l'action collective, mais aussi sur les propos tels qu'ils sont tenus par les professionnels.

Sur le plan méthodologique, nous mobilisons des données recueillies dans 4 départements, via trois types de méthodes: la constitution de groupes de réflexion dans chaque département autour des enjeux liés au GÉVA Sco, I'observation de 9 situations d'instructions de dossiers de la première réunion à l'école au passage en CDAPH pendant un an et la réalisation de 72 entretiens auprès des acteurs clés de chaque situation.

Dans un premier temps, nous présenterons les raisons qui ont prévalu à la création du GÉVA Sco. Ensuite, nous présenterons les registres de cohérence et les enjeux relatifs aux équipes de suivi de scolarisation et aux équipes éducatives, pour enfin analyser ceux relatifs aux équipes pluridisciplinaires d'évaluation.

Nous verrons que ces registres représentent une certaine homogénéité, même si la présentation de bilans extérieurs à l'école, la prise en compte du contexte territorial et un rapport différent à la parole des familles donnent lieu à des évaluations différentes.

\section{À L'ORIGINE, UNE VOLONTÉ DE RECONFIGURER L'ÉVALUATION}

Créé à l'origine par la Caisse nationale de solidarité pour l'autonomie et la Direction générale de l'enseignement scolaire, le GÉVA Sco a pour vocation d'harmoniser les pratiques d'évaluation en imposant un processus unique synthétisé par l'idée que les équipes de suivi de scolarisation et les équipes éducatives sont le lieu du constat de la difficulté de l'élève et que les équipes pluridisciplinaires sont le seul espace d'évaluation (CNSA, 2012).

Autrement dit, les équipes se tenant à l'école ne doivent ni faire d'évaluation, ni faire de préconisation, notamment en termes de besoins de compensation ou d'orientation. De l'autre côté, la législation permet et encourage la présence d'acteurs de terrain dans les équipes pluridisciplinaires, puisqu'il s'agit d'évaluer des situations où le contexte local a un impact important.

Dans ce contexte, le système actuel tend à produire des inégalités territoriales (Amara et al., 2012), notamment en termes d'attribution d'aide humaine ou de reconnaissance

3. Commission des droits et de l'autonomie des personnes handicapées. 
administrative du handicap. Si le GÉVA Sco impose l'idée que l'évaluation se joue uniquement en MDPH, afin d'harmoniser les pratiques d'évaluation, d'autres dynamiques constituent un maillage évaluatif plus complexe.

Dans les observations, il n'a pas été rare de voir que les équipes pluridisciplinaires laissaient aux équipes de suivi de scolarisation le soin d'évaluer la répartition des temps de scolarisation dans les décisions de temps partagé entre école et établissement spécialisé. S'il s'agit de favoriser la scolarisation en milieu ordinaire, il est, dans le même geste, reconnu que ce genre d'évaluation ne peut pas être réalisé de manière distanciée de la situation.

Si, avec le GÉVA Sco, il s'agit de recentrer chaque type d'équipe à ses missions premières, il apparaît difficile de retirer toute forme d'évaluation à des professionnels du secteur médico-social ou de l'enseignement dont l'une des missions est de mesurer des performances ou des capacités, dans le but de transmettre des savoirs. Cette complexité renvoie à l'articulation nécessaire entre suivi personnalisé et harmonisation des pratiques évaluatives.

Cette articulation entre connaissance du terrain et mise à distance des acteurs existe également en CDAPH, faisant de cette articulation le véritable fil conducteur du processus décisionnel. En réunissant représentants des services de l'État, d'associations et élus, il ne s'agit plus de croiser les regards, mais de croiser intérêts privés et publics. Dans le même temps, la description des situations tend largement à personnaliser la situation de l'élève, à partir de critères non seulement scolaires ou médicaux, mais aussi personnels, relatifs au parcours de vie ou à la situation familiale.

\section{DES CONCEPTIONS DU BESOIN ET DE L'AUTONOMIE FORTEMENT DIFFÉRENCIÉES}

Dès lors, il apparaît que les différences dans l'évaluation s'opèrent sur les manières de concevoir l'autonomie et les besoins. Les groupes de réflexion réalisés dans les quatre départements étudiés, réunissant parents, membres de la MDPH et professionnels de l'Éducation nationale et du médico-social, ont permis de faire émerger trois conceptions divergentes.

Tout d'abord, à partir d'une vision médico-juridique, les membres de l'équipe pluridisciplinaire expriment toujours le même principe réglementaire, suivant un processus systématisé, en vue du passage de la proposition en CDAPH: existence avérée de déficience, retentissement sur la vie de la personne, présence ou non de solution dans le droit commun et proposition de compensation le cas échéant. Ensuite, certains enseignants considèrent que l'autonomie et l'évaluation des besoins se mesurent à l'aune des résultats scolaires. Cette conception de la mesure est présente, tant chez des enseignants que chez des agents administratifs de la MDPH vus en entretien. Selon une professeure principale au collège, l'autonomie se mesure "par rapport au rendu du travail, qu'on lui demande déjà, par rapport aux questions qu'il peut nous poser en cours [...] on va travailler davantage sur du chiffré à un moment donné ". Même si l'inclusion scolaire relève du "bien-être ", les enjeux entourant les résultats scolaires priment. Cette conception rejoint d'ailleurs celle à l'œuvre dans une partie de la littérature scientifique (Deslandes, Potvin, Leclerc, 2000). 
Enfin, la troisième approche se fonde sur le socle commun de compétences et sur l'idée que la socialisation se construit à l'école. II s'agit de saisir toutes les dimensions relatives à la vie sociale dans le cadre scolaire, afin de concrétiser à terme la citoyenneté. Cela revient à penser les besoins de manière beaucoup plus large, rappelant en cela les différentes grilles constituées afin de catégoriser les besoins fondamentaux (Maslow, 1943). Dès lors, les questions relatives au bien-être de l'enfant priment sur les notes. Si l'autonomie ne se joue pas uniquement à partir des résultats scolaires, l'évaluation s'ancre nécessairement dans cette dimension. Les résultats scolaires détermineront le choix d'orientation et à long terme l'insertion professionnelle.

Si le GÉVA Sco reprend des éléments relatifs au GÉVA ${ }^{4}$ pour mesurer l'autonomie, le document intègre également les apprentissages à partir de l'idée de performance. Chaque domaine d'activité doit être côté $A, B, C$ ou D, afin d'identifier les tâches réalisées avec ou sans aide ou lorsqu'elles sont non réalisées.

$\mathrm{Si}$, dans les observations en équipe pluridisciplinaire, le GÉVA Sco est utilisé de manière quasiment systématique, l'évaluation est réalisée avec plusieurs documents. Ainsi, dans un département, la MDPH demande aux enseignants du premier degré de remplir une fiche construite à partir du socle commun de compétences. À partir du secondaire, il est systématiquement demandé le bulletin de notes. Enfin, il n'est pas rare que des écrits non corrigés de l'élève soient demandés.

Au-delà des oppositions discursives constituées lors des groupes de réflexion, l'évaluation pluridisciplinaire semble s'être construite en prenant en compte les trois dimensions évoquées.

\section{À L'ÉCOLE, PROJET, PERFORMANCE ET CAPACITÉS SUPPOSÉES PRIMENT}

Les réunions ayant lieu dans les écoles - équipe de suivi de scolarisation et équipes éducatives, ayant trait au projet de l'élève, à son niveau scolaire, à ses capacités supposées, ainsi qu'au contexte local, nous avons pu identifier trois manières de les mener.

La première consiste à aiguiller les échanges autour du projet de l'élève, des difficultés rencontrées et des moyens mis en œuvre et à demander. Ce type de réunion s'est présenté lorsque la situation paraissait évidente à tous les acteurs, notamment en termes de difficultés de l'élève et de besoins à compenser.

La deuxième manière consiste à orienter la discussion, afin de trouver un consensus. Ces situations se présentent lors de potentiels conflits. Ainsi, un enseignant référent a mené une équipe après un premier échec de scolarisation. Pour lui, il s'agissait d'abord de réussir cette deuxième tentative en posant que "l'école est accueillante ", en favorisant les échanges entre la nouvelle enseignante et une enseignante spécialisée et surtout en "rassurant les parents", afin de commencer un accompagnement relevant du dévoilement du handicap (Ebersold, 2007).

La troisième façon de mener les équipes renvoie à un engagement relevant du respect des injonctions institutionnelles et réglementaires, en lien avec l'école

4. Guide d'évaluation de l'autonomie; il s'agit du document réglementaire en vigueur dans les MDPH. 
inclusive. Cette manière de faire se fonde sur une forme de mise à distance des difficultés enseignantes et une volonté d'imposer une scolarisation la plus proche possible du milieu ordinaire. Dans le cas d'un élève scolarisé en temps partagé entre école et établissement spécialisés, l'enseignante et la chef d'établissement se sont interrogées sur l'utilité d'une orientation à temps plein dans un dispositif spécialisé, argumentant que l'élève avait peut-être une déficience intellectuelle plutôt que des troubles du comportement.

Dès lors, l'enseignante référente a argumenté qu'un élève déjà orienté dans un établissement spécialisé dans les troubles du comportement ne pouvait pas être orienté dans un dispositif spécialisé dans la déficience intellectuelle, de par la nature de son trouble. Suite à une proposition d'orientation Segpa ${ }^{5}$, l'équipe enseignante s'était inquiétée du niveau de l'élève, l'orientation n'étant possible qu'avec " un niveau CE2 ». Une solution fut trouvée au moment où le coordinateur de l'établissement spécialisé a proposé de faire réaliser un bilan, même si, pour lui, le jeune n'avait pas de déficience intellectuelle. Au final, le bilan a permis de mettre d'accord le collectif: cet élève n'avait pas de déficience intellectuelle.

Ainsi, le déroulement des équipes de suivi de scolarisation se fait en fonction de dynamiques relevant de la qualification du trouble, du niveau de performance de l'élève et du projet scolaire en jeu. Selon la manière dont ces trois éléments s'articulent, la réunion ne sera pas menée de la même manière, sachant que la scolarisation en milieu ordinaire demeure une injonction forte.

\section{La temporalité comme élément central du jugement scolaire}

En ce sens, les difficultés sont présentées, en fonction d'un projet scolaire. II s'agit de déterminer le type d'orientation envisageable ou le type d'aide que pourrait apporter une notification de la MDPH. Si les aménagements pédagogiques sont envisagés sous l'angle de solutions déjà apportées, ils sont souvent présentés comme des solutions insuffisantes et temporaires.

In fine, ces dimensions sont toujours envisagées à partir de la temporalité. Tout élément évoqué se fait en rapport avec le passé ou l'avenir, notamment en termes de suivi spécifique au regard des progrès dans un temps donné et en lien avec les échéances d'orientation. L'élève est alors présenté en fonction de ses difficultés et de ses réussites.

Ainsi, en équipe de suivi de scolarisation, la grille d'autonomie du GÉVA Sco, souvent remplie avant la rencontre, est lue selon deux axes. Le premier renvoie à la performance dans les apprentissages, c'est-à-dire la capacité effective d'apprendre à apprendre. L'autre axe renvoie à un descriptif se fondant sur les grands domaines d'activité du GÉVA (hygiène corporelle, tâches et exigences en relation avec la scolarité, etc.) et sur les compétences scolaires ou sociale telles qu'envisagées par les enseignants. Sur le plan des compétences sociales, de nombreuses remarques liées à l'attitude, tant en classe que dans la cour de récréation, à l'hygiène, à l'intimité ou au corps, renvoient autant à la socialisation primaire qu'à la posture d'élève, voire de futur jeune adulte intégrable au monde du travail.

5. Section d'enseignement général et professionnel adapté. 
Suite à ces constats relevant de l'évaluation construite sur l'idée d'une norme à atteindre, des conseils sont prodigués aux parents sur l'hygiène, les devoirs à la maison ou sur l'éducation de l'enfant. Cette tendance est d'autant plus forte que les compétences des autres professionnels comme les éducateurs, renvoient au travail social.

La mention fréquente de "l'anxiété " ou des "angoisses" de l'élève ne renvoient pas seulement à l'idée, comme cela a été mentionné dans de nombreux entretiens, que "le plus important est que l'enfant se sente bien", mais aussi qu'en état de "bien-être ", les élèves sont plus performants, en termes de concentration, de mémorisation, de compréhension et d'apprentissages effectifs.

À ces registres liés à la performance s'ajoutent ceux relatifs à l'aide à apporter pour favoriser l'inclusion, compenser les manques de l'élève ou ceux de l'école. Ainsi, il n'est pas rare que les difficultés de l'équipe pédagogique ou de l'enseignant puissent trouver une solution dans une forme de compensation individuelle, l'environnement n'étant pas adapté à l'élève. Concernant les difficultés de ce dernier, les types d'aides donnés dans le travail scolaire ou la nécessité que l'enfant a de s'appuyer systématiquement sur l'adulte sont autant d'arguments pour arriver à la demande d'aide humaine, d'orientation ou de matériel adapté.

\section{Occultation d'éléments explicatifs et dilemmes enseignants}

Comme l'enfant est d'abord vu comme un élève, les situations sociales et familiales sont peu évoquées. Par exemple, pour une élève en dispositif inclusif, l'enseignante a listé les difficultés scolaires en pointant que cette année, elle faisait " des choses moins difficiles que l'an dernier", alors que cette dernière trouvait les exercices plus durs. Ce n'est qu'une fois les éléments scolaires présentés que la mère en a expliqué la raison: "son frère a failli mourir cette année ".

Dans le contexte de l'équipe de suivi de scolarisation, les éléments familiaux sont éludés, alors qu'ils peuvent avoir un pouvoir explicatif important. N'entrant pas dans les catégories de pensée en jeu, ces éléments ne sont pas pris en compte pour présenter l'élève. Cette forme catégorielle a pour effet de construire un fil directeur particulier.

Ainsi, lorsque le trouble a été clairement identifié, il n'est jamais mentionné, bien que les solutions envisagées se fassent en lien avec cette connaissance. Dans le cas d'un jeune enfant autiste de 4 ans, l'enseignante spécialisée explique comment le faire progresser sur le plan de la communication, cette difficulté apparaissant comme majeure: "il faut l'obliger à nous solliciter [...] par exemple, nous, on met les objets en hauteur ". L'enseignant référent prend souvent la parole afin de saisir le comportement relationnel de l'enfant, mais aussi ses capacités à entrer, in fine, dans les apprentissages.

Ces questionnements relatifs à un suivi personnalisé se heurtent à des enjeux qui dépassent le seul cas de l'enfant. Ainsi, suite à la mise en coprésence de cet enfant avec d'autres de son âge, des actes de violence sont apparus. Alors que l'enfant commençait à s'exprimer verbalement, il a été décidé de ne pas accroître le temps de scolarisation tant que ce problème ne serait pas résolu. Pour l'enseignant référent, " avec l'idée que ça nous met vraiment en contact avec lui, on a remarqué 
[...] qu'il venait vraiment plus vers nous [...] mais c'est pour le moment sous une forme vraiment assez désagréable pour tout le monde [...] c'est la manière qui n'est pas adaptée ".

Au-delà des actes, les uns et les autres retiennent l'aspect envahissant d'un tel comportement. Ainsi, lors de l'entretien, I'enseignante spécialisée résume le dilemme des enseignants en milieu ordinaire: " oui, alors faut-il prioriser l'enfant à besoins particuliers? Faut-il prioriser le groupe? Faut-il mettre des priorités ou pas? [...] ils ne savent plus, vraiment s'ils doivent prioriser. On parle beaucoup d'inclusion, ce qui est très bien, comme ils disent, mais en même temps, ils se disent: et les autres enfants?"

À l'inverse, dans le cas d'une élève de $3^{e}$, malentendante et bénéficiant d'une auxiliaire de vie scolaire, les résultats scolaires vont motiver la professeure principale et le chef d'établissement à proposer la $2^{\text {nde }}$ générale, alors que le père, l'aide humaine et l'orthophoniste insisteront pour une orientation en $2^{\text {nde }}$ professionnelle. Si, lors de l'équipe de suivi de scolarisation, les arguments se fondent d'abord d'un côté sur les notes et de l'autre sur les capacités supposées au regard de la déficience auditive, d'autres éléments sont à considérer.

Ainsi, I'aide humaine explique: "l'échec, elle apprécie pas! Ils ont fini par comprendre que c'était pas quelque chose d'envisageable [...] Elle a certainement un niveau très bon pour aller en bac pro mais elle va être plus à l'aise".

Surtout, le choix pour la $2^{\text {nde }}$ professionnelle intervient dans un contexte familial où les études ne sont pas une priorité, l'insertion professionnelle étant perçue comme possible via des formations plus courtes. En ce sens, d'autres dimensions interviennent dépassant le niveau scolaire ou la nature du trouble. Pourtant, de par le cadre institutionnel, ils ne peuvent être envisagés en équipe de suivi de scolarisation.

\section{À LA MDPH, DE L'ADMINISTRATION DE LA PREUVE À LA MESURE DE LA PERFORMANCE}

In fine, si l'équipe de suivi de scolarisation est le lieu des constats, il est d'abord un espace d'évaluation spécifique se centrant sur des éléments relatifs aux capacités d'apprentissage de l'élève. Qu'il s'agisse du GÉVA Sco, du socle commun de compétences ou des résultats à proprement parler, il s'agit de mesurer les performances de l'élève et ses capacités à acquérir des connaissances. En ce sens, certains éléments ne sont pas pris en compte par les professionnels, permettant dans le même temps aux parents d'investir ces réunions. Par la présentation d'éléments relatifs à la vie de famille, ils peuvent défendre l'intérêt de leur enfant.

La question du constat des difficultés renvoie donc d'abord à une forme d'évaluation professionnelle à partir de critères mettant à distance des éléments explicatifs extérieurs à l'école. L'enjeu n'est pas ici de saisir la situation d'un point de vue global, mais bien de porter un regard de spécialiste.

En équipe pluridisciplinaire, si le travail ne poursuit pas les mêmes objectifs, un certain nombre de principes, d'enjeux et de registres présidant à l'évaluation se rapprochent de ceux déjà évoqués. Néanmoins, l'évaluation étant corrélée par plusieurs bilans professionnels, celle-ci prend une autre forme. 


\section{Le faisceau d'indices comme principe d'administration de la preuve}

II apparaît tout d'abord que l'évaluation en MDPH se fonde sur la lecture d'un certain nombre de documents. Le premier d'entre eux, le projet de vie, est systématiquement lu. Suite à cela, les éléments médicaux, le compte rendu psychologique, le bilan de l'orthophoniste ou de tout autre spécialiste sont présentés. Dans un autre registre, un bilan social est aussi lu. Enfin, les éléments scolaires sont généralement présentés à partir de plusieurs documents dont la personne référente a fait une synthèse.

Dans le cadre réglementaire qui est le leur, les membres de l'équipe d'évaluation doivent d'abord vérifier l'existence d'un trouble avéré. En outre, les orientations en établissement spécialisé sont possibles uniquement sous certaines conditions. Ainsi, une référente scolarisation explique: "on nous demande par exemple une entrée en Itep pour un enfant qui a des troubles du comportement mais il n'y a pas eu d'évaluation psychométrique d'effectuée [...] une orientation en Itep ne peut se faire que si on est sûr que l'enfant n'est pas dans la déficience cognitive. "

Les aspects psychologiques et médicaux apparaissent comme prépondérants, mais, à l'inverse, le GÉVA Sco sert dans plusieurs départements à déterminer le taux d'incapacité: "il nous permet d'évaluer justement le retentissement de la situation de handicap, savoir si [...] on est en situation de handicap. Après, il nous permet d'évaluer le retentissement en terme de taux d'incapacité, est-ce qu'on est dans des troubles modérés, des troubles importants [...] c'est un des éléments, il y contribue beaucoup." "

Ainsi, la question du diagnostic renvoie aux enjeux réglementaires, imposant une nécessaire administration de la preuve. Les cas où les différents bilans sont contradictoires, tout en utilisant des termes similaires sont particulièrement éclairants. Dans un cas particulier, bilans orthophonique et phonologique se contredisaient sur le fait qu'il y ait retard de langage, tandis que le bilan psychologique ne reconnaissait aucune déficience. Pour autant, les éléments scolaires, en particulier le GÉVA Sco, faisaient état de problèmes de mémorisation, de retard du langage et de la parole. Surtout, même si l'orthophoniste notait qu'un "verrou avait sauté ", ce suivi était mis en place depuis trois ans, prouvant aux yeux des membres de l'équipe pluridisciplinaire que pour cet enfant, " on est dans le champ du handicap ». Dans le même temps, les difficultés de diagnostic ont poussé cette équipe à proposer de "la faire entrer dans le champ du handicap, avec un taux en cours d'investigation ", tout en refusant l'aide humaine demandée.

Il apparaît aussi que lorsque le certificat médical met en avant " un contexte social et culturel défavorisé ", voire des "carences et des négligences éducatives ", plus qu'une déficience, le cas est exclu du champ du handicap, d'autant plus lorsque le point de vue enseignant rend compte d'un " manque d'investissement des parents dans le travail à domicile ".

Le rôle endossé par les membres de l'équipe pluridisciplinaire les contraint à exprimer des formes de jugement de l'élève et des acteurs concourant à sa scolarisation. Néanmoins, ils ne sont pas jugés sur le même mode. Ainsi, les parents le sont au regard de leur responsabilité légale, renvoyant à une forme de jugement existant depuis longtemps dans le travail social ou à l'école, et dont 
nous trouvons trace dans les équipes de suivi de scolarisation. Néanmoins, les membres de ces équipes ayant à croiser plusieurs éléments, ils ne jugent pas l'action parentale sur les mêmes critères.

Leur vision est d'abord modelée en fonction du degré " d'acceptation » ou de " déni du handicap " qu'ils accordent aux familles, auprès desquelles il s'agit de faire un travail " d'accompagnement ». Dans certains cas, le taux d'incapacité est attribué selon le rapport que les parents entretiennent avec le handicap de leur enfant. II s'agit alors de les pousser à " prendre conscience ", mais aussi de leur "laisser le temps".

Le croisement des éléments permet aussi aux membres de l'équipe pluridisciplinaire de prendre du recul, dans certains cas, sur le regard des travailleurs sociaux et des enseignants. Ainsi, une famille ayant beaucoup de difficultés à mettre en place des soins tels que l'orthophonie s'est opposée à la proposition d'orientation en établissement médico-social de l'équipe d'évaluation, voulant que leur enfant reste en dispositif inclusif.

Dans cette situation, la famille a argumenté que leur enfant commençait à progresser. Des échanges entre l'enseignante et la référente scolaire de la MDPH s'en sont suivis. Suite à la réception d'un GÉVA Sco très détaillé, la référente scolaire, porteuse de la proposition d'orientation en établissement médico-social, a changé d'avis lorsqu'elle a lu que cet enfant " commence à entrer dans les apprentissages fondamentaux ", qu'il a une "meilleure organisation du travail, dans la relation aux autres " et qu'il prend des initiatives. Dès lors, la référente scolaire s'est voulue "plus nuancée", reconnaissant que les " progrès actuels pourraient lui permettre d'aller en dispositif inclusif au collège " à terme et argumentant qu' " on est face à un enfant qui a l'air de commencer à évoluer $"$.

S'agissant des professionnels, il en va autrement. Les membres de l'équipe pluridisciplinaire tendent à produire un discours dépersonnalisé et environnemental. Ainsi, lors de la description des résultats scolaires d'un jeune, il a été expliqué que l'institut où il suivait sa formation adaptait "énormément " les enseignements, à tel point qu'il y avait risque après l'obtention du CAP de ne pouvoir intégrer le monde du travail. L'équipe envisage l'action des professionnels au regard de leurs contraintes institutionnelles ou par rapport aux spécificités de l'établissement au sein duquel cette action est produite.

Cela montre par ailleurs que les membres de l'équipe n'ont pas de prise sur la réalité sociale qu'ils évaluent. Ainsi, un jeune garçon de 12 ans, inscrit en Segpa à temps plein, bénéficiait en même temps d'une aide humaine individualisée. Or, la CDA avait, à l'origine, notifié une scolarisation en milieu ordinaire avec AVS-i et des temps en Segpa uniquement pour le français sans AVS-i, ce jeune ayant des troubles du langage et étant étranger. Dans le même temps, la commission départementale d'orientation avait orienté le jeune en Segpa.

Le collège étant " un gros établissement, ils n'ont pas pu " mettre en œuvre la notification de la MDPH. Se rendant compte de l'impossibilité de réorienter ce jeune en $4^{e}$ générale, malgré de "très bons résultats ", l'équipe finit par proposer aide humaine mutualisée et poursuite en Segpa, pour envisager à terme une fin de prise en charge. 


\section{Une évaluation scolaire fondée sur la capacité à apprendre}

La question de l'orientation étant fondamentale, les échanges autour de la scolarisation sont extrêmement importants et structurent une grande partie des bilans reçus par la MDPH. Quels que soient les professionnels, nous retrouvons les mêmes éléments de langage.

Les termes anxiété ou angoisse sont autant utilisés par des psychologues libéraux ou scolaires que par des enseignants. Si cet usage ne renvoie pas aux mêmes conceptions, cela dénote un déplacement du terme dont le sens a changé au cours du temps, par ces différents glissements. Ce déplacement permet de relever deux principes.

Le premier est celui de langage commun voulu par le législateur (CNSA, 2010; CNSA, 2012). Le deuxième est celui de traduction (Latour, 1989; Bureau, 2013). Ainsi, pour fonder l'évaluation professionnelle, chaque corps de métier s'empare du champ lexical de l'autre. II ne s'agit plus seulement de prendre des éléments relatifs à la psychanalyse comme cela fut le cas dans d'autres instances par le passé (Castel, 1973), mais bien d'intégrer plusieurs champs disciplinaires.

À partir de cette multiplicité, l'équipe pluridisciplinaire fonde son approche sur deux versants, I'un sur la capacité à apprendre et l'autre sur la socialisation, ce second versant ayant pour objectif de permettre à l'enfant d'entrer dans les apprentissages. Selon une chef de service de MDPH, pour un cas particulier: " au départ, on était plutôt sur la socialisation, c'est ce qu'on nous donnait comme information, et puis essayer de rentrer dans les apprentissages. "

Dans ce cadre, le niveau scolaire est étudié au travers de plusieurs documents. Selon la même chef de service, "les bulletins de note, ça nous permet de savoir comment se situe l'élève aussi, par rapport à la classe et puis, quelles sont ses compétences scolaires. Et puis, les écrits [...] sur les troubles du langage ça nous permet de voir si la communication écrite est entravée ". Avec le GÉVA Sco, "je pense que par rapport à l'éligibilité à la prestation de compensation [...] C'était des éléments beaucoup plus scolaires avant. Là, on prend peut-être un peu de distance, un peu plus ".

In fine, les registres de cohérence s'articulent tous autour d'un langage commun fondé sur un champ lexical s'appuyant sur les mondes de la psychologie, de l'éducation et de savoirs professionnels paramédicaux. Pour autant, c'est le registre scolaire, afin de déterminer le niveau de l'élève qui prend une place plus importante. Ainsi, la référente scolarisation de l'équipe joue ici un rôle central: " c'est toujours par rapport au niveau de classe que l'on est [...] Lire en CP qu'est-ce que ça veut dire? Lire en CE2 qu'est-ce que ça veut dire? [...] Lire en $3^{e}$, qu'est-ce que ça veut dire? [...] À chaque fois, on se reporte au niveau de classe. "

Dans ce cadre, le GÉVA Sco a une place importante pour prendre en considération de manière appuyée la capacité à acquérir des connaissances, notamment en poussant à évaluer le repérage dans le temps, des lieux, la motricité tant fine que globale ou l'autonomie dans les gestes de la vie quotidienne.

Ainsi, les questions relatives à l'attitude sont évoquées, notamment lorsque l'enfant "ne soutient pas le regard", faisant référence en cela au trouble diagnostiqué dont on trouve trace dans le comportement. De même, les termes relatifs à la 
gentillesse, au courage, au respect, au bonheur à venir à l'école et leurs inverses, sont systématiquement évoqués. Mais surtout, les éléments relatifs à la fatigabilité, au temps de concentration, à la lecture, à l'écriture et au calcul sont présentés pour évaluer la capacité à performer.

Par ailleurs, les autres professionnels faisant leur bilan en fonction des enjeux liés à l'école, une orthophoniste écrit: " amélioration des 2 voies de lecture, enrichissement du stock lexical orthographique, amélioration de la combinatoire phonème/graphème, augmentation du stock de vocabulaire, amélioration du langage oral ». Suivant la même logique, les écrits des enseignants présentent les difficultés scolaires au regard du trouble comme l'expression d'un "retard " en lien avec trouble du langage. Ainsi, les aspects positifs de l'élève sont vus sous ce même angle de performance dans l'apprentissage, au regard du trouble: "Capable d'acquisition, peut progresser à condition d'aller à son rythme et de tenir compte de son handicap".

Enfin, la posture d'élève est systématiquement interrogée, toujours dans le but d'entrer dans les apprentissages. Ainsi, la « posture d'élève a progressé mais pas les apprentissages ". Dans ce cas, la référente scolaire: "je pose la question, éventuellement établissement médico-social à temps partagé avec un dispositif inclusif? Parce qu'il n'est pas non plus en refus du scolaire ".

\section{CONCLUSION: PLUSIEURS APPROCHES POUR DÉTERMINER LE DEGRÉ DE PERFORMANCE DE L'ÉLÈVE}

L'évaluation des besoins renvoie à une approche particulière. Si elle a été qualifiée de globale (Barreyre, Peintre, 2004), il n'en demeure pas moins qu'elle n'existe que par rapport à des objectifs et un projet, dont l'inclusion n'est que le moyen et les différents dispositifs la mise en œuvre. Nous assistons ici à une traduction visant à agréger des intérêts professionnels et publics divergents, parfois contradictoires, tout en correspondant aux dispositifs existants et aux représentations véhiculées par la notion même d'accès aux droits, ayant consacré une définition du handicap fondée sur l'existence avérée d'une déficience ou d'une pathologie durable.

S'il apparaît que l'évaluation des besoins ne renvoie pas à un besoin de service, mais bien à un besoin éducatif (Houchot, Robine, 2007; Ebersold, Detraux, 2012), il s'agit de s'interroger sur ce que les différents acteurs entendent par ce terme. D'un côté, il s'agit de donner la " capacité effective des individus à déterminer leur propre règle de conduite et à s'y tenir dans le cadre de leur environnement social " (Duvoux, 2009). D'un autre côté, I'action publique poursuit l'objectif d'améliorer la qualité de vie des personnes (Detraux, Di Duca, 2003). En partant des capacités et des aptitudes de l'enfant en milieu scolaire, l'évaluation des besoins revient à s'interroger sur les moyens menant à l'autonomie, une des contreparties du droit social (Dufour et al., 2003). Pour autant, si en équipe de suivi de scolarisation les enjeux relatifs à l'évaluation semblent se fonder exclusivement sur le projet scolaire au regard d'une performance, en équipe pluridisciplinaire, les évaluateurs agrègent différentes formes d'évaluation.

L'utilisation du GÉVA Sco, du bulletin de notes, du socle commun de compétences et des écrits non corrigés, revient non seulement à croiser les regards, mais 
surtout à trouver un dénominateur commun à l'étude de ces documents relevant de conceptions différentes du sens à donner à la scolarisation et à l'éducation. Dès lors, il s'agit de saisir les capacités effectives à apprendre, dans un contexte social et culturel plus général, dans lequel l'accès à l'emploi demeure l'enjeu, alors que ces élèves courent le risque d'être perçus comme inemployables (Ebersold, 2001). En ce sens, l'amélioration de la qualité de vie passe par un accroissement de I'autonomie et donc par l'adaptation à la nouveauté, ce dernier item revenant très régulièrement dans l'évaluation. Cette capacité à s'adapter est alors relative au trouble de l'enfant, mais aussi et peut-être surtout à la faisabilité d'un projet. En ce sens, le projet de l'élève ne peut se construire qu'en lien avec une réalité qui serait à la fois interne à la personne et liée avec les projets de formation délivrés par les établissements (Boutinet, 2008).

In fine, pour les acteurs, qu'ils soient à l'école ou en MDPH, il s'agit d'articuler des principes, en apparence, contradictoires: inclusion et performance.

\section{Références}

Akrich, M., Rabeharisoa, V. (2012). L'expertise profane dans les associations de patients, un outil de démocratie sanitaire, Santé Publique, 24(1), 69-74.

Amara, F., Vigneron-Meleder, H., Delauber, J.-P., Caraglio, M. (2012). L'accompagnement des élèves en situation de handicap. Les prescriptions: état des lieux-propositions. Rapport de I'IGAS, de I'IGEN et de I'IGAENR, 2012-162.

Barreyre, J.-Y., Peintre, C. (2004). Évaluer les besoins des personnes en action sociale. Enjeux, méthodologie, outils. Paris: Dunod.

Bateson, G. (2008). Vers une écologie de I'esprit. Paris: Le Seuil.

Blanc, P. (2002). Compensation du handicap: le temps de la solidarité. Rapport d'information au nom de la commission des affaires sociales du Sénat.

Boltanski, L., Thevenot, L. (1991). De la justification. Les économies de la grandeur. Paris: Gallimard.

Boutinet, J.-P. (2008). Vulnérabilité adulte et accompagnement de projet. Un espace paradoxal à aménager. In J.-P. Boutinet, N. Denoyel, G. Pineau, J.-Y. Robin. Penser l'accompagnement adulte. Ruptures, transitions, rebonds. Paris: PUF.

Bureau, M.C. (2013). La traduction de la demande d'aide sociale: les cas du handicap et de l'insertion des jeunes. Revue française d'administration publique, 145, 175-188.

Bureau, M.-C., Rist B. (2013). La rationalisation gestionnaire contre la subjectivation: l'exemple de l'aide aux personnes handicapées. In C. Bellot, Le travail social à I'heure de la nouvelle gestion publique. Cloisonnement des pratiques ou nouvelles opportunités. Québec: PUQ.

Deslandes, R., Potvin, P. Leclecrc, L. (2000). Les liens entre l'autonomie de I'adolescent, la collaboration parentale et la réussite scolaire. Revue canadienne des sciences du comportement, 32(4), 208-217. 
Castel, R. (1973). Le psychanalysme: I'ordre psychanalytique et le pouvoir. Paris: Maspero.

Castel, R. (1991). Savoirs d'expertise et production des normes, In F. Chazal, J. Commaille, Normes juridiques et régulation sociale. Paris: LGDJ.

CNSA. (2010). Rapport sur l'appropriation du GÉVA. Guide d'évaluation des besoins en compensation des personnes handicapées.

CNSA (2012). Manuel GÉVA-Sco. Scolarisation.

Detraux, J.-J., Di Duca, M. (2003). Méthodologie d'analyse des besoins des élèves et de développement d'un projet d'accompagnement adapté. Rapport de recherche [en ligne] www.enseignement.be/download.php?do_id=1860\&do_check

Dufour, P., Boismenu, G., Noël, A. (2003). L'aide au conditionnel: la contrepartie dans les mesures envers les personnes sans emploi en Europe et en Amérique du Nord. Montréal: Presses universitaires de Montréal.

Duvoux, N. (2009). Les assistés peuvent-ils être autonomes? Sociologie compréhensive des politiques d'insertion. Lien social et politiques, 61.

Ebersold, S. (2001). La naissance de l'inemployable. Ou l'insertion aux risques de l'exclusion. Rennes: PUR.

Ebersold, S. (2007). Parents et professionnels face au dévoilement du handicap. Dires et regards. Ramonville Saint-Agne: Érès.

Ebersold, S. Detraux, J.-J. (2013). Scolarisation et besoin éducatif particulier: enjeux conceptuels et méthodologiques d'une approche polycentrée. Alter, European Journal of Disability Research, 7(2), 102-115

Epstein, S. (1995). The construction of lay expertise: AIDS activism and the forging of credibility in the reform of clinical trials. Science, Technology \& Human Values, 20(4), 408-437.

Fassin, D. (2000). La supplique. Stratégies rhétoriques. Annales. Histoire, sciences sociales, 55(5).

Fassin, D. (2010). La raison humanitaire. Paris: EHESS.

Houchot, A., Robine, F. (2007). Les livrets de compétences: nouveaux outils pour l'évaluation des acquis. IGEN, rapport $\mathrm{n}^{\circ} 048$.

Latour, B. (1989). La science en action. Paris: La Découverte.

Lesain-Delabarre, J.-M. (2006). Considérer les parents comme les premiers acteurs de l'éducation d'un enfant handicapé: vers un nouveau dogme ? La nouvelle revue de l'adaptation et de la scolarisation, 34, 71-88.

Maslow, A. (1943). A Theory of Human Motivation. Psychological Review, 50, 370-396.

Tessaire, N. Parentalité et situations de handicap, réflexions d'un professionnel. Reliance, 4, 26, 63-67.

Thery, I. (2005). Expertises de service, de consensus, d'engagement: essai de typologie de la mission d'expertise en sciences sociales. Droit et société, 60, 311-329. 